\title{
Simulating Haptic Feedback of Abdomen Organs on Laparoscopic Surgery Tools
}

\author{
Shirani M. Kannangara ${ }^{1 *}$, Eranga Fernando ${ }^{1}$, Sumudu K. Kumarage ${ }^{2}$, Nuwan D. Nanayakkara ${ }^{1}$ \\ ${ }^{1}$ Department of Electronic \& Telecom. Eng., Faculty of Engineering, University of Moratuwa, Sri-Lanka. \\ 2 Department of Surgery, Faculty of Medicine, University of Kelaniya, Kelaniya, Sri-Lanka. \\ * Corresponding author. +94-714434536; email: mangalika@ent.mrt.ac.lk \\ Manuscript submitted March 10, 2015; accepted May 18, 2015 \\ doi: 10.17706/ijbbb.2015.5.3.193-201
}

\begin{abstract}
Minimally invasive surgeries (MIS) such as laparoscopic procedures are widely used for many types of abdomen surgeries because of its numerous advantages over open surgeries. They require very high level of skills of surgeons acquired through experience. The best and the safest way of getting hands on experience is the computer simulation or virtual reality (VR). The VR surgical simulators have a great potential to revolutionize the training paradigm of surgical interns. The haptic feedback plays as equally as visual feedback to provide realistic environment to trainees. In this paper, we present a method incorporate hapitics on VR simulator. A software procedure is developed using the Libraries of Open Haptic Toolkit along with the Open GL graphic libraries to implement three basic haptic ranges: soft, mild(firm) and hard into organ models. The feedback of the expert surgeons in the field was obtained to model the organs rather than measuring mechanical properties of soft tissues due to practical limitations. A commercially available six Degrees of Freedom (DoF) position sensing and three DoF force feedback haptic devices are used to implement the interface.
\end{abstract}

Key words: Force feedback, haptic, laparoscopic surgeries, virtual reality.

\section{Introduction}

Minimally Invasive Surgeries are considered today to be a better way of performing many abdomen surgeries due to a variety of benefits such as short hospital stays, fast recovery time, less post operative care, fewer complications and a low rate of infections over open surgeries [1]. Although patients are benefitted from this new technology, during laparoscopic procedures surgeons have several challenges like loss of depth perception of the images of organs in the area of interest produced by the endoscopic camera, the fulcrum effect and the reduced haptic feedback for tool-tissue interaction. The indirect visualization and indirect manipulation of tools disturb the hand-eye coordination of the user [1], [2]. Complexities due to lack of experience sometimes force surgeons to convert the laparoscopic procedure to conventional surgeries mainly. Therefore, a proper training is essential for surgical interns before assisting in the actual surgeries [3], [4].

\section{Previous Related Work}

The available training methods for laparoscopic surgeries are categorized as box trainers [5], Virtual Reality (VR) simulators [5]-[7] and Augmented Reality (AR) surgical simulators [5], [8], [9]. The box trainers are equipped with physical models such as synthetic or fabric material or sometimes are cadavers 
[10]. Since the system uses real laparoscopic instruments to interact with organ models, the trainee will experience the interaction forces through the tool handle while training. However, this feedback is different from the natural feedback provided by live surgeries. On the other hand, this system does not provide objective evaluation which is essential to evaluate trainee's skills before transferring to operating room. Organ models which need frequent replacement and thus quite expensive, and therefore, box trainers are not widely available in the healthcare system around the world.

The general consensus is that VR simulators provide an immediate solution for laparoscopic surgery [9] training over traditional box trainers [10]. VR simulators provide objective evaluation of trainee performance but most of the simulators lack the feeling of tool- tissue interaction forces as provided in box trainers with physical objects. Majority of work carried out has emphasized that well designed computer simulation system enables the trainee surgeon to improve and enhance psychomotor skills for laparoscopic surgeries. In most VR simulators, haptic solutions are far behind compared to visualization because of the complexity of the procedures [5]. Many researchers have reported that haptic feedback is essential for VR simulators to improve the fidelity of the simulator [1], [2], [7], [9], [11], [12].

Augmented Reality (AR) is an advanced training paradigm of VR simulators. The AR combines the virtual reality with the physical world [5]. AR simulators provide the objective assessment of trainee performance as VR simulators in addition to the haptic feedback provided by box trainers [5]. Even though the realistic haptic feedback is fundamental to the VR simulator, majority of researchers have concluded that it would be a very critical task [1], [12] as haptics needs simultaneous input/output mechanism which involves a large number of touch receptors [13], [14]. It has been found that the required haptic update rate for realistic haptic feedback is at least $1000 \mathrm{~Hz}$ [15]. However this is highly challenging compared to visual refresh rate which is about $30 \mathrm{~Hz}$ [13], [16]. Other key factor is the availability of proper organ force models. As the mechanical properties of biological tissues are very complex [1], [2], [7], [15], [16] and ethical issues are involved, the development of deformable organ models is rather critical. Therefore, overcoming this lack of facility in VR simulators is a highly challenging active research area for medical simulators.

There are numerous VR simulators currently available for MIS training [7], [13]. Each simulator consists of different training modules for trainees to learn many basic skills of laparoscopic procedures such as cholecystectomy and gynecology [13]. None of the VR simulators equipped with proper organ models and they do not provide enough feedback to tool-tissue interaction as experienced in actual surgeries [13]. Therefore, the existing training methods do not fulfill trainee requirement [7], [12].

The organ models are a core component of a VR training simulator to display accurate displacement for pulling and pushing forces [17]. Incorporating real force properties on VR organ models is a very difficult problem faced by many researchers around the world [6], [8], [12]. In this paper, we propose a novel approach to incorporate haptics to virtual organ models to simulate laparoscopic surgery tool interaction with tissues. As an initial step of the procedure, we implemented three basic force ranges: soft, mild, and hard force feedback through tool handles on abdomen organ models. We believe that, the simulated organ models can provide an interactive training environment to trainee surgeons.

\section{Methodology}

VR simulators have been developed for a wide range of procedures to find solutions for the difficulty in haptic perception during training. The development of proper organ models is a highly challenging task in this procedure [6], [12], [16]. Researchers have used CT (Computed Tomography), MRI (Magnetic Resonance Imaging) of Visible Human data set of abdomen (VHP abdomen) [18] to develop virtual models of anatomical organs [2], [8], [19]. In this study, we used the color Cryosection data set of Visible Human Project of the National Library of Medicine, USA to generate virtual models of abdomen organs due to its 
image quality and high resolution over CT and MRI data [2]. In the first phase of this project, virtual models of five abdomen organs such as liver, gallbladder, bowel, stomach and skin were developed using VTK and ITK open source software [17]. Fig.1 shows the organ moles developed using color cryosection data of the Visible Human Project of the National Library of Medicine. Kinesthetic feedback plays a major role in laparoscopic surgeries and it is extremely difficult to gain a proper training without this feedback. Therefore, the hardware interface system must be capable of providing interaction forces along with the correct position and orientation: roll, pitch and yaw of the tool-tip. Furthermore, the system needs high precision simultaneous input/output mechanism to provide the feeling of real-life surgery.

\subsection{Previous Implementation}

In this section we summarize our previous work on developing a hardware interface to incorporate haptics into a laparoscopic surgical simulator [17]. The system incorporated with two optical incremental encoders to measure 2-DoF (yaw and pitch). An optical displacement sensor and a high resolution potentiometer were used for the measurement of the other two DoF (roll and insertion). The system was capable to provide spatial location information of the tool tip in the work space in addition to the force feedback feeling in all three axes for tool-tissue interaction up to some extent. Even though the system is a low cost and less precise method, it can provide high resolution at an acceptable price. Fig. 2 shows the mechanical model of hardware interface designed for the VR simulator.

The movement of the laparoscopic tool is mapped with the virtual interface. Therefore, the physical tool tip position of the laparoscopic tool will be the same as the tool position in the virtual environment. However, there were many challenges in designing the hardware interface which gives four DoF. The system requires light weight, durability and resistance to the deformation. Aluminum is lightweight and durable but the resistance to deformation was not adequate. Therefore, the different parts of the system were designed with aluminum together with nylon. The shaft which connected to encoders undergoes deformation that was very difficult to avoid. This leads to incorrect readings in the encoder. The braking system consists of an electromagnet which can activate accordingly when the tool tip touches the organ models in the virtual environment. The braking system constrains the tool movement when it collides with virtual organ models. The virtual environment calculates the forces needed for haptic feedback and transmits to the braking system.

The amount of braking force depends on the type: soft, firm (mild) or hard of the virtual organ models. For instance, less braking force is required to identify soft tissues compared to hard tissues. Because of light weight and smaller in size, braking can be controlled by the induced current to the system.

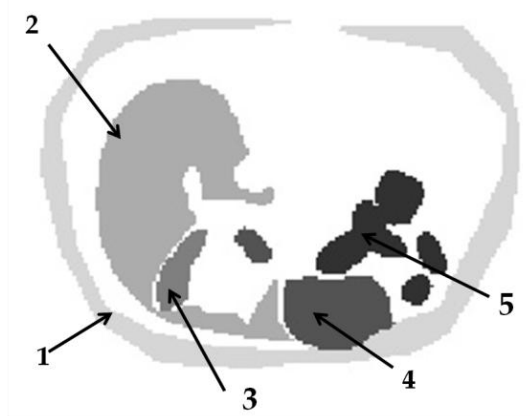

Fig. 1. Axial view of abdomen organ models: 1 : skin, 2: liver, 3: gallbladder, 4: stomach, 5: bowels.

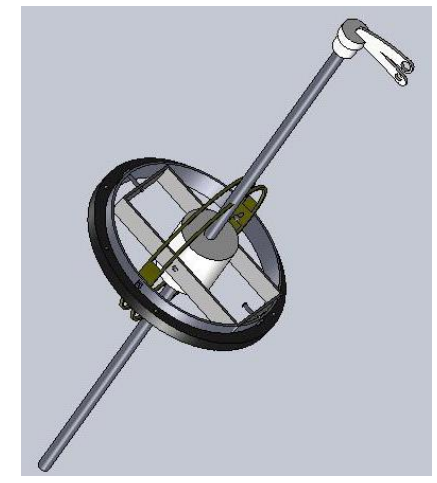

Fig. 2. Design of the mechanical model of hardware interface. 


\subsection{Proposed Implementation}

Due to the delay in the braking system and limitations of accuracy, the previous system did not meet the users' experience for force feedback up to the required level. Because of these drawbacks and limitations, our next approach is to develop the hardware interface through which the surgical residents can gain skills in a very realistic manner.

Very precise haptic devices are commercially available today and they vary greatly in the degrees of freedom they offer, the force and torque they can apply, the size of their workspace, the shape of the end effectors and probably, in price [13]. To feel both forces as well as torques, it requires six degrees of force feedback but typically this is not easy to provide due to many technical difficulties. In our study, we have selected the Phantom Omni haptic device [20] to provide six-Degrees of Freedom positional sensing along X, $\mathrm{Y}$ and $\mathrm{Z}$ axis together with the tool orientation (roll, pitch and yaw) in work space. In addition to this positional sensing of the laparoscopic tool, the device provides three DoF force feedbacks to the simulator.

To reflect actual feedback for tool tissue interaction, the organ models should contain the actual tissue properties. However, developing organ models with actual tissue properties is a rather complex issue and thus very little work is carried out to incorporate real organ properties [16]. Therefore, we introduce a novel method to implement force feedback for interaction forces. A software procedure is developed using the Libraries of Open Haptic Toolkit along with the Open GL graphic libraries to implement three basic haptic ranges: soft, mild/firm and hard into organ models. The proposed method allows the user to change the tissue properties such as, damping constant, stiffness and friction of organ models. The user can feel the interaction of virtual tools with the said organ models while taking tool navigation information from the same tool. The proposed VR interface can separate the organ models initially into three force ranges: soft, mild/firm and hard and the user can experience the force feedback through the haptic interface for the laparoscopic tool as shown in Fig. 3.

Soft tissues are very complex and their mechanical properties are not properly known. We hypothesis that if the organ models are developed with different normalized stiffness values, then they could provide natural feeling to the user. The stiffness is normalized by the device maximum stiffness value, which is 2.31 $\mathrm{N} / \mathrm{mm}$ of the selected device. For this research, we obtained the feedback of the experts in the field. They interacted with the virtual organ models through the haptic interface by changing the stiffness of tissues. The feedback for tool tissue interaction with organ models was collected by changing the normalized stiffness of organ models. We used this data to average normalized stiffness values of the organs in three categories representing soft, mild and hard properties. We then used the calculated normalized stiffness values to implement haptic properties of virtual organ models.

\section{Results}

The feedback was obtained three times from expert surgeons and twice from senior registrars and registrars for each organ model. The feedback obtained from registrars spreads over a large range and therefore, variation is relatively large compared to surgeons. Therefore, we did not use the feedback obtained from registrars and considered only the feedback of expert surgeons to implement soft, mild and hard force ranges to the virtual organs. Fig. 4 shows the combined data obtained from experienced surgeons.

The normalized stiffness of liver is quite high compared to other organs as shown in Fig. 4. The liver is considered to be a firm (mild) organ and the other four organs: gallbladder, stomach, bowels and vessels are considered as relatively soft organs. Thus to determine soft and firm force ranges, the average values are considered. Table 1 summarizes the average normalized stiffness for each organ with their standard deviation. 


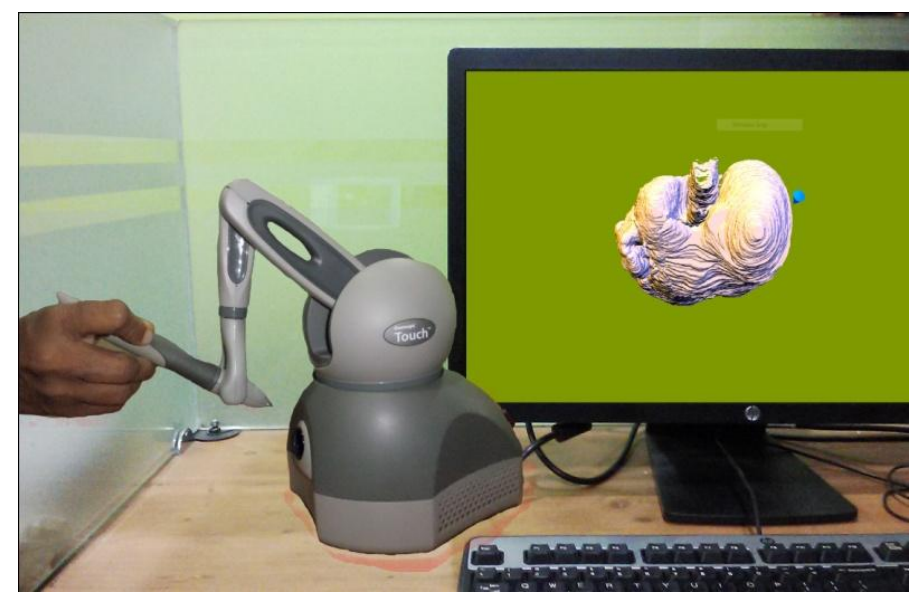

Fig. 3. Hardware interface to the proposed VR Simulator.

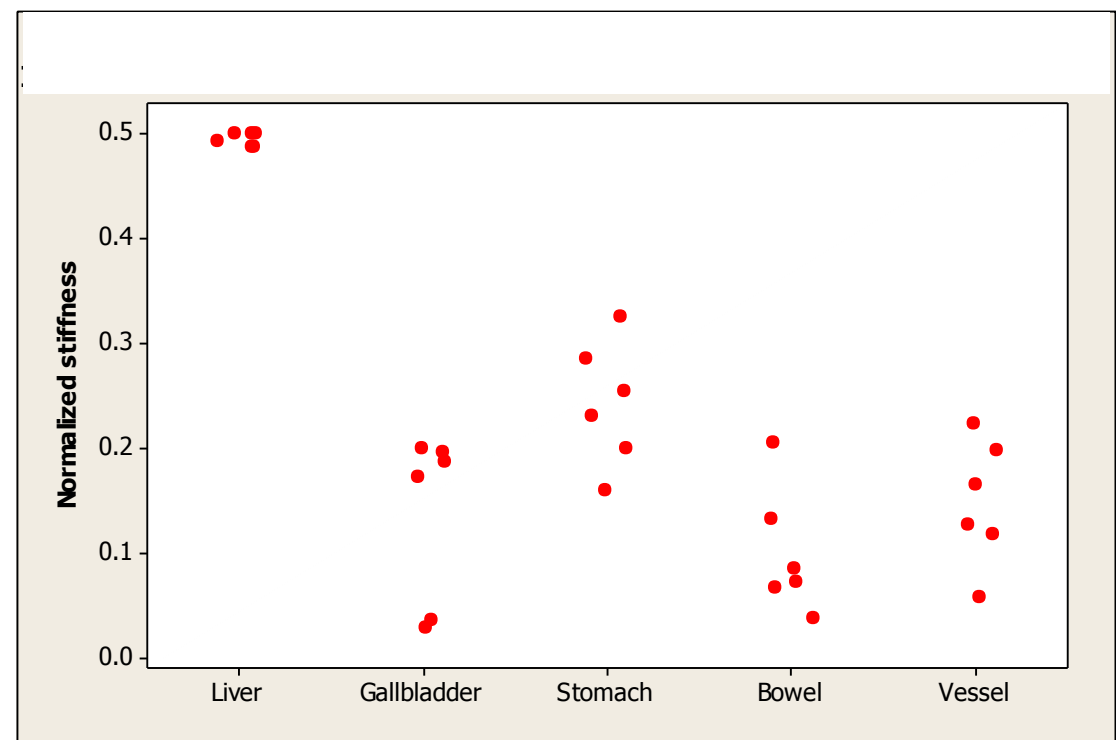

Fig. 4. Individual stiffness value plot of liver, gallbladder, stomach, bowels and vessel feedback for all tries of expert surgeons.

Table 1. Average Normalized Stiffness Values of Organs with Their Standard Deviation

\begin{tabular}{ccc}
\hline \hline Organ & Mean & Std. deviation \\
\hline liver & 0.49 & 0.01 \\
gallbladder & 0.14 & 0.08 \\
stomach & 0.24 & 0.06 \\
bowel & 0.10 & 0.06 \\
vessel & 0.15 & 0.06 \\
\hline \hline
\end{tabular}

To determine a fair normalized stiffness value for soft feeling, the average of mean values of each soft organ shown in Table 1. is considered. Accordingly, we estimated the most appropriate normalized stiffness to provide soft and mild/firm feelings to the user. Table 2 summarizes the calculated normalized stiffness and estimated stiffness values that we can assign to simulate soft and mild/firm feelings. By considering the difference of the normalized stiffness vales corresponding to soft and mild feelings along with our experience, we propose a normalized stiffness value of 0.83 to the simulator to experience the hard feeling.

We conducted experiments for evaluating the experience of soft, mild/firm and hard force ranges of the organ models. As an initial step of evaluation of the simulator, we have used three identical cubes with soft, 
firm and hard force ranges. In the testing procedure, ten identical cubes with different force ranges are randomly generated and the trainee has to touch the organ models and determine whether the model is soft, firm or hard. We have done the preliminary evaluation with a non specialized group. For that we have selected ten research students in our biomedical laboratory and conducted the procedure as follows. In the first round, the trainees were given a good training through which they studied three force ranges properly. Immediately after the training, the Test 1 is carried out. The second round was started at least two hours later after the first test for the same group. Test 2 was performed without any training, only with their past experience. Immediately after the Test 2, Test 3 is carried out after giving an another training session. Results obtained from each trainee are tabulated in Table 3.

Table 2. Estimated Stiffness Values for Three Basic Haptic Properties of Tissues

\begin{tabular}{ccc}
\hline \hline Tissue property & Normalized stiffness & Estimated stiffness N/mm \\
\hline soft & 0.16 & 0.37 \\
mild/firm & 0.49 & 1.13 \\
hard & 0.83 & 1.92 \\
\hline \hline
\end{tabular}

Table 3. Percentage of Accuracy of Ten Participants of Identifying Soft, Firm/Mild and Hard Organ Property

\begin{tabular}{|c|c|c|c|c|c|c|c|c|c|c|c|c|}
\hline Test round & ipant & 1 & 2 & 3 & 4 & 5 & 6 & 7 & 8 & 9 & 10 & $\begin{array}{l}\text { Average } \\
\text { with } \\
\text { standard } \\
\text { deviation }\end{array}$ \\
\hline First round & Test 1 & 90.0 & 70.0 & 90.0 & 70.0 & 70.0 & 70.0 & 70.0 & 100.0 & 80.0 & 40.0 & $75.0 \pm 16.5$ \\
\hline Second & Test 2 & 90.0 & 90.0 & 80.0 & 80.0 & 100.0 & 70.0 & 70.0 & 80.0 & 70.0 & 60.0 & $79.0 \pm 11.4$ \\
\hline round & Test 3 & 100.0 & 100.0 & 100.0 & 80.0 & 90.0 & 100.0 & 70.0 & 100.0 & 90.0 & 90.0 & $92.0 \pm 9.8$ \\
\hline
\end{tabular}

According to results in Table 3, the average accuracy of selecting correct organ models increases with the increase of training sessions while the standard deviation is decreased. Therefore, our simulator supports trainee students to categorize organ models basically into soft, firm/mild and hard. Table 4 summarizes the probability of selecting correct organ property in each test. According to the results in Table 4, with the training sessions, probability of selecting a soft model is highly accurate compared to other two types.

Table 4. Probability of Identifying Correct Organ Property

\begin{tabular}{cccc}
\hline \hline Organ property & Test1 & Test2 & Test3 \\
\hline soft & 0.92 & 1.00 & 1.00 \\
mild/ firm & 0.65 & 0.75 & 0.89 \\
hard & 0.63 & 0.63 & 0.88 \\
\hline \hline
\end{tabular}

\section{Conclusion and Future Directions}

We have proposed a novel method to simulate haptic feedback of abdomen organ models for tool tissue perception using a commercial haptic device. The organ models were generated in the three basic force ranges soft, mild and hard which considered to be the minimum requirement. The process mainly involved in collecting force data directly from the experienced surgeons for estimating feedback forces. The simulated organ models are capable of providing force feedback in all three dimensions. The generated organ models could enhance the existing VR laparoscopic training systems by incorporating natural feelings through the tool handles. The system is mainly developed with the open source software with a Phantom Omni haptic device.

The mechanical behavior of biological tissues is extremely complex and viscoelastic. Soft tissues exhibit 
nonhomogeneous, anisotropic and nonlinear behavior [15], [16]. Properties also change with the age and sex. Furthermore, properties change with the in vivo and ex vivo state of measurements. High computational power and fast haptic update rate are also key issues for this kind of simulation [13], [16]. Therefore, majority of researchers have mentioned the difficulty of developing a real time simulator for laparoscopic surgery training. Very little work is done on real organ force models. Thus, we have followed a new approach to integrate force feedback into organ models. We have developed a software procedure which can support to change the normalized stiffness of organ models. The force feedback experienced for tool tissue interaction in actual surgeries was obtained with the support of experienced surgeons. The surgeons who were involved in this study have surgical experience of above seven years and gave us very good feedback to develop reasonably accurate organ models in the simulator.

We are in the process of assessing the precision of the simulated organ models with experts in the field. In future, we hope to improve the organ models by simulating deformation and grasping forces.

\section{Acknowledgment}

This project is supported by the HETC (Higher Education for the 21st Century) project of the Ministry of Higher Education in Sri Lanka, funded by the World Bank and the Senate Research Grant (SRC/LT/2011/14), University of Moratuwa. Furthermore, authors sincerely appreciate U.S. National Library of Medicine for the image data from the Visible Human Project.

\section{References}

[1] Westebring, E. P., Putten, V. D., Goossens, R. H. M., Jakimowicz, J. J., \& Dankelman, J. (2008). Haptics in minimally invasive surgery-a review. Minimally Invasive Therapy, 17, 3-16.

[2] Basdogan, C., Edef, M., Harders, M., \& Wesarg, S. (2007). VR-based simulators for training in minimally invasive surgery. IEEE Computer Socitey, 27(2), 54-66.

[3] Zhang, J., Huang, W., Zhou, J., Yang, T., Liu, J., et al. (2011). Gallbladder modeling and simulation in laparoscopic cholecystectomy. Proceeding of the IEEE Conference on Industrial Electronics and Applications, 49(20), 128-131.

[4] Hiemstra, E., Terveer, E. M., Chmarra, M. K., Dankelman, J., \& Jansen, F. W. (2011). Virtual reality in laparoscopic skills training: Is haptic feedback replaceable? Minimally Invasive Therapy, 179-184.

[5] Botden, S. M. B. I., \& Jakimowicz, J. J. (2009). What is going on in augmented reality simulation in laparoscopic surgery? Surg. Endosc., 23(8), 1693-1700.

[6] Dubois, P., Thommen, Q., \& Jambon, A. C. (2002). In vivo measurement of surgical gestures. IEEE Transactions on Biomedical Engineering, 49, 49-54.

[7] Dankelman, J. (2008). Surgical simulator design and development. World J. Surg., 32, 149-155.

[8] Linte, C. A., White, J., Eagleson, R., Guiraudon, G. M., \& Peters, T. M. (2010). Virtual and augmented medical imaging environments: Enabling technology for minimally invasive cardiac interventional guidance. IEEE Reviews in Biomedical Engineering, 3, 25-47.

[9] Rosen, J. Hannaford, B., Richards, C. G., \& Sinanan, M. N. (2001). Markov modeling of minimally invasive surgery based on tool/tissue interaction and force/torque signatures for evaluating surgical skills. IEEE Transactions on Biomedical Engineering, 48, 579-591.

[10] Megali, G., Sinigaglia, S., Tonet, O., \& Dario, P. (2006). Modeling and evaluation of surgical performance using hidden markov models. IEEE Transactions on Biomedical Engineering, 53, 1911-1919.

[11] Hamza-Lup, F. G., Seitan, A., Popovici, D. M., \& Bogdan, C. M. (2012). Medical simulation and training: "Haptic Liver". The seventh International Conference on Virtual Learning (pp. 27-33).

[12] Meijden, O. A. J. V. D., \& Schijven, M. P. (2009). The value of haptic feedback in conventional and 
robot-assisted minimal invasive surgey and virtual reality training: A current review. Surg. Endosc., 23(6), 1180-1190.

[13] Coles, T. R., Meglan, D., \& John, N. W. (2011). The role of haptics in medical training simulators: A survey of the state of the art. IEEE Transactions on Haptics, 4(1), 51-66.

[14] Satava, R. M., \& Jones, S. B. (1998). Current and future applications of virtual reality in medicine. Proceedings of the IEEE, 86, 484-488.

[15] Pezzementi, Z., Ursu, D., Misra, S., \& Okamura, A. M. (2008). Modeling realistic tool-tissue interactions with haptic feedback: A learning-based method. Proceedings of Symposium on Hhaptic Interfaces for Virtual Environments and Teleoperator Systems (pp. 209-215). USA: Reno, Nevada.

[16] Liu, A., Tendick, F., Cleary, K., \& Kaufmann, C. (2003). A survey of surgical simulation: Applications, technology and education. Presence Teleoperators \& Virtual Environments, 12, 599-614.

[17] Kannangara, S. M., Ranasinghe, S. C., Kumarage, S. K., \& Nanayakkara, N. D. (2014). Hardware interface for haptic feedback in laparoscopic surgery simulators. IEEE Region 10 Technical Symposium (pp. 376-380). Malaysia: Kuala Lumpur.

[18] Spitzer, V., Ackerman, M. J., Scherzinger, A. L., \& Whitlock, D. (1996). The Visible human male: A technical report. Journal of the American Medical Informatics Association, 3(2), 118-130.

[19] Aucar, J. A., Groch, N. R., Troxel, S. A., \& Eubanks, S. W. (2005). A review of surgical simulation with attention to validation methodology. Surg. Laparoscopic Endosc Percutan Tech., 15(2), 82-89.

[20] Sensable Technologies (1999-2008). Open Haptics Toolkit, version 3, printed in USA.

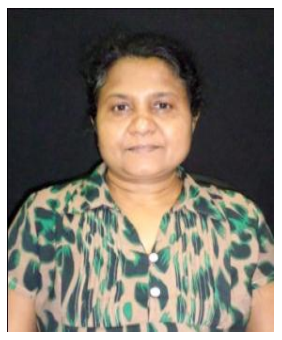

Shirani Mangalika Kannangara was born in Kalutara on 6th March 1967. She obtained her B.Sc. in physical science degree from University of Colombo, Sri Lanka with a second class (upper division) honors, in 1996. She is currently serving as a lecturer in the Division of Electrical and Electronic Engineering Technology, Institute of Technology, University of Moratuwa, Sri Lanka. Furthermore, she is doing her postgraduate studies in the Premium Biomedical Laboratory of University of Moratuwa. She is interested in medical imaging and medical image processing related research such as surgical simulators.

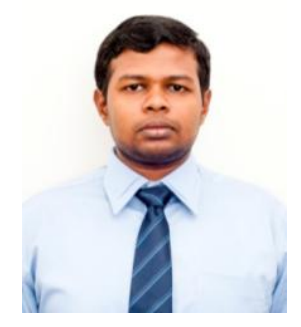

Eranga Fernando was born in 1988. He obtained his B.Sc (Hons) in the Electronic and Telecommunication Engineering of University of Moratuwa, Sri Lanka in 2013. He is currently working as a lecturer at the Department of Electronics and Telecommunication Engineering of University of Moratowa and also working in the UAV Research Laboratory of the University. He has worked as a research assistant in the Premium Biomedical Laboratory of University of Moratuwa. He has given courses on robotics, industrial robotics and electronics. His research interests include robotics, control systems and unmanned aerial vehicles. 


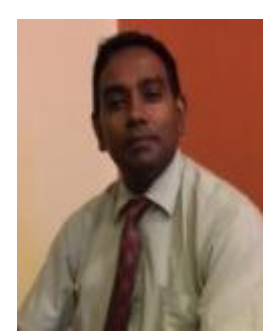

Sumudu Kalum Kumarage was born in Colombo on 21st November 1965. He obtained the MBBS Colombo in 1993, MS surgery - Colombo in 2000, FRCS Ed in 2001. He is currently the head of the Department of Surgery and the senior lecturer in surgery at the Teaching Hospital Colombo - North University of Kelaniya. He served as a consultant surgeon in Base hospital Ampara from 2004 to 2006. His main research interests are laparoscopic and colorectal surgery. He is also a member of the College of Surgeons of Sri Lanka and a fellow of the Royal College of Surgeons of Edinburgh.

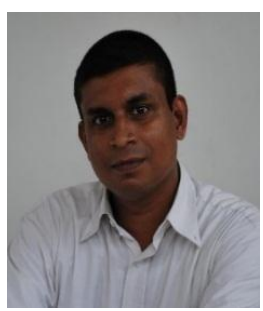

Nuwan Dayananda Nanayakkara received his B.Sc. engineering degree in electronics \& telecommunication from University of Moratuwa, Sri Lanka with first class honors, in 1999. He obtained his M.E.Sc. degree in electrical \& computer engineering at the University of Western Ontario, London, Ontario, Canada in 2003, and obtained his Ph.D. degree at the same university in biomedical engineering in 2008. He is currently serving as a senior lecturer in the Department of Electronic \& Telecommunication Engineering at University of Moratuwa, Sri Lanka. His research focuses on using engineering principles to solve problems in medicine and biology, or biomedical engineering. 